\title{
Plasma Diagnostic Reflectometry
}

Bruce I. Cohen

Bedros B. Afeyan

John C. Garrison

Thomas B. Kaiser

Neville C. Luhmann, Jr.

Calvin W. Domier

Albert E. Chou

S. Baang

February 26, 1996






\section{DISCLAIMER}

This document was prepared as an accoount of work sponsored by an agency of the United States Government. Neither the United States Government nor the University of California nor any of their employees, makes any warranty, express or implied, or assumes any legal liability or responsibility for the accuracy, completeness, or usefulness of any information, apparatus, product, or process disclosed, or represents that its use would not infringe privately own rights. Reference herein to any specific commercial products, process, or service by trade name, trademark, manufacturer, or otherwise, does not necessarily constitute or imply its endorsement, recommendation, or favoring by the United States Government or the University of California. The views and opinions of authors expressed herein do not necessarily state or reflect those of the United States Government or the University of California, and shall not be used for advertising or product endorsement purposes.

This report has been reproduced directly from the best available copy.

Available to DOE and DOE contractors from the Office of Scientific and Technical Information

P.O. Box 62, Oak Ridge, TN 37831

Prices available from (615) 576-8401, FTS 626-8401

Available to the public from the National Technical Information Service

U.S. Department of Commerce 5285 Port Royal Rd.

Springfield, VA 22161 


\section{DISCLAMERR}

Portions of this document may be illegible in electronic image products. Images are produced from the best available original document. 


\title{
Plasma Diagnostic Reflectometry
}

\author{
Bruce I. Cohen, Bedros B. Afeyan, John C. Garrison, and Thomas B. Kaiser \\ Lawrence Livermore National Laboratory, University of California \\ Livermore, California 94550
}

\author{
Neville C. Luhmann, Jr., Calvin W. Domier, Albert E. Chou, and S. Baang \\ Department of Applied Science, University of California at Davis \\ Livermore, California 94550
}

\begin{abstract}
Theoretical and experimental studies of plasma diagnostic reflectometry have been undertaken as a collaborative research project between the Lawrence Livermore National Laboratory (LLNL) and the University of California Department of Applied Science Plasma Diagnostics Group under the auspices of the Laboratory Directed Research and Development Program at LLNL. Theoretical analyses have explored the basic principles of reflectometry to understand its limitations, to address specific gaps in the understanding of reflectometry measurements in laboratory experiments, and to explore extensions of reflectometry such as ultra-short-pulse reflectometry. The theory has supported basic laboratory reflectometry experiments where reflectometry measurements can be corroborated by independent diagnostic measurements.
\end{abstract}




\section{Introduction}

Microwave reflectometry is an important plasma diagnostic method that has been used to study the density profile of the ionosphere and increasingly in magnetic fusion experiments (Mazzucato, 1975; Simonet,1985; Hubbard, et al., 1987; Baang, et al., 1990; Doyle, et al., 1990; Manso, 1993). The aim is to make spatially localized measurements of the plasma density profile including turbulent density fluctuations. Reflectometry is based on the principle that an electromagnetic wave launched into a plasma reflects from a welldefined layer in the plasma depending jointly on the wave frequency and the density profile for an ordinary $(O)$ mode (and both the density and the magnetic field profile for an extraordinary (X) mode). By varying the frequency of the incident microwave field and measuring the change in the time delay (group delay), monotonic density profiles can be reconstructed. Furthermore, a modulation of the plasma dielectric caused by a density fluctuation (or a magnetic fluctuation) along the ray path will produce a modulation of the phase and amplitude of the probing microwave. This in turn allows one to extract information on the fluctuations from the reflected microwaves.

Reflectometry has become an important diagnostic in fusion plasmas for several reasons. It has the advantage that it gives a localized measurement of the plasma density at a location inside the plasma without having to insert a material probe into the plasma, and the diagnostic is typically non-perturbative. Reflectometry requires a single viewing chord and minimal vacuum access in contrast to other techniques such as interferometry and Thomson scattering. Furthermore, collective scattering provides poor spatial resolution and does not appear to be well suited to the next generation of magnetic fusion experiments. Because turbulent fluctuations degrade the confinement of heat and particles in tokamaks, a reliable measurement of the fluctuations with good spatial resolution is an important prerequisite to understanding the nature of the turbulence and in learning to control it. There are numerous examples in the literature of the use of reflectometry to measure fluctuations. Reflectometry has been used by N. Luhmann's group and his collaborators to measure localized, long-wavelength fluctuations in the Texas Experimental Tokamak (TEXT) and the DIII-D tokamak at General Atomics (Peebles, et al., 1990). Reflectometry has also been used to measure density fluctuations in the Tokamak Fusion Test Reactor (TFTR) at Princeton (Paul, et al., 1992).

Although the use of reflectometry has grown steadily, the theoretical understanding of reflectometry is surprisingly limited. There have been few calculations, and those have been one-dimensional almost exclusively (Mazzucato and Nazikian, 1991; Chou, et al., 1992; Hutchinson, 1992). In the last few years, there have been at least two efforts that : 
included two-dimensional effects (Irby, et al., 1993; Hansen, et al., 1993) by groups other than our own. The work of Irby, et al. (1993) is a direct numerical solution of the wave equation for O-mode propagation in the presence of a density fluctuation in two dimensions. Hansen and co-workers (1993) analytically calculated the scattering of microwaves by a filamentary density perturbation in an underdense plasma using Green's function techniques. Neither of these two-dimensional studies, nor any of the onedimensional calculations, are able to resolve the localization paradox of many reflectometry meaurements, in which fluctuation correlation lengths are inferred that are shorter than the width of the last Airy lobe of the probing microwaves near the reflection layer. This localization paradox, as well as additional discrepancies between experimental reflectometry data and theory (enhanced phase sensitivity observed and discrepant dependence on fluctuation wavenumber (Chou, et al., 1992)), and the general lack of an understanding of reflectometry motivated our coordinated theoretical and experimental reflectometry research under the auspices of LDRD labwide funding in FY94-95.

The objectives of our reflectometry research were to (1) provide a comprehensive theory basis for reflectometry by extending previous one-dimensional calculations and by developing two- and three-dimensional models using both analytical means and a suite of codes, (2) continue and extend basic reflectometry experiments, comparing them to theoretical analysis and simulations, and determining the feasibility of making accurate measurements of density fluctuations in fusion experiments, and (3) provide theory for and conduct advanced reflectometry experiments (realized with moderate-pulse and ultra-shortpulse reflectometry).

We have addressed the localization paradox of reflectometry signals directly and have found mechanisms that are good candidates to resolve the paradox (Afeyan, 1994). We have delineated parameter regimes where either the Born approximation or the WKBJ method used in analytical calculations may be valid by comparing to direct numerical solutions of the wave equations. We identified two- and three-dimensional effects that can give rise to the discrepancies between one-dimensional theory and the basic reflectometry experiments conducted by Luhmann's group. Our calculations have established the general conditions that ensure the reliability of reflectometry to measure density fluctuations. We have demonstrated the feasibility of ultra-short-pulse reflectometry (Domier, et al., 1995) for future use in important fusion experiments. As part of this research program, two graduate students in Prof. Luhmann's UC Davis Plasma Diagnostic Group have received training and guidance in plasma physics, mathematical physics, and computational physics in support of their laboratory plasma experimental work. 


\section{Code Development and Modeling}

A suite of reflectometry codes has been developed by this project. HELM1D is a one-dimensional Helmholtz equation solver for monochromatic reflectometry that has been extended to accommodate exponentially tapered profiles and to model the extraordinary (X) mode as well as ordinary (O) mode. SOFTSTEP 1D and 2D are time-dependent codes using a slowly varying envelope approximation which has been tested and applied to the basic laboratory plasma reflectometry experiment. A three-dimensional massively parallel version of SOFTSTEP has been begun which includes new coherence and correlation function diagnostics. We have also developed a suite of fully time-dependent onedimensional (Cohen, et al. 1995) and two-dimensional O-mode and X-mode codes that have been used to model laboratory ultra-short-pulse reflectometry experiments.

SOFTSTEP 1D was used to show the fundamental difference in effects produced by fluctuations at the cut-off layer that produce amplitude and phase changes in the reflected signal and fluctuations at Bragg resonance that produce only a phase shift in the reflected signal. This had a bearing on interpretations of the data from the laboratory plasma reflectometry experiment. HELM1D was used to study further Bragg resonance effects on reflectometry and the departures of the scattering from the description given using the first Born approximation (Afeyan, et al., 1995; Chou, et al., 1995).

Ultra-short-pulse reflectometry was studied with the suite of full-wave one- and two-dimensional O-mode and X-mode codes. Density-profile reconstructions were modeled, and their robustness in the presence of large-amplitude density perturbations was studied to determine operating limits on the reconstructions. With X-modes, magnetic profiles were reconstructed in addition to density profiles. Furthermore, it was demonstrated that Bragg resonance effects due to density perturbations could be identified in the reflected signals in addition to and distinct from the signals from cut-off layers that were used for the profile reconstruction, thus providing data on the density perturbations. A more robust and reliable method for determining the dispersion of the group delays used in the reconstructions was implemented in the suite of ultra-short-pulse codes based on a windowed fast Fourier transform (a spectral method). The spectral method is analogous to the laboratory techniques, allowed us to accurately reconstruct a wider variety of plasma density profiles, and was reliable in the presence of a higher amplitude density perturbation than was the case using the zero-crossing method in our earlier calculations. We have also included an embedded conducting surface in our two-dimensional computations to model a waveguide launcher and detector more closely resembling the experiments, and have obtained good reconstructions using the windowed FFT method. We have explored : 
resolution issues relevant to profile reconstructions in the experiments which give guidance on how many frequency channels should be used in the detectors.

Figures 1-11 provide examples from our modeling effort. Figures 1 and 2 present results from one-dimensional computations of X-mode reconstructions of density profiles with a nonuniform magnetic field and reconstructions of the magnetic field with a known density profile. Figure 3 illustrates the use of ultra-short-pulse reflectometry to deduce characteristics of both the overall density profile and fluctuations producing return signals from Bragg resonance. Two-dimensional computations of ordinary mode ultra-short-pulse reflectometry are shown in Figs. 4, 5, and 6. Density-profile reconstructions based on group delay vs. frequency data obtained from both zero-crossing and windowed Fourier transform of the reflected electric field compare well for simple density profiles, but the windowed Fourier method (which is what is employed in the laboratory experiments) is far superior for a stepped density profile although stringent demands on resolution are incurred. Figures 7, 8, and 9 display the results of two-dimensional reconstructions of $\mathrm{O}$ mode reflectometry with a model for the waveguide launching structure included in the computation (the launching structure is modeled by an open-ended structure with conducting walls embedded in the computational mesh) and with and without superposed density perturbations. The windowed Fourier method is quite robust and can accommodate density perturbations of significant relative amplitude (Fig. 8). When the number of frequency channels drops below $\sim 10$, the resolution of the profile reconstruction degrades significantly (Fig. 9). Figures 10 and 11 show results from our analytical studies of Bragg resonance using the Born approximation, one of whose applications is to understanding the effects of density fluctuations on reflectometry signals (Afeyan, 1994; Afeyan, et al., 1995; Chou, et al., 1995). Figure 10 shows the validity of the Born approximation in calculating the scattering phase shift as a function of $\left(\omega_{0} L / c\right)^{2 / 3} \delta \mathrm{n} / \mathrm{n}_{0}$. The HELM1D code was used to make the comparisons. A direct comparison of the analytical expression for the scattering phase shift and that produced by HELM1D is presented in Fig. 11. The transition of the phase shift out of the Born approximation regime can be seen clearly.

\section{Reflectometry Experiments}

\section{A. Controlled Laboratory Filament Discharge Plasma Experiments}

Numerous improvements were made to the laboratory plasma experiment (Figure 12), both in terms of reflectometer signal launching structures as well as in improved diagnostics. Among the many diagnostic improvements to the laboratory plasma experiment is the ability to make detailed electromagnetic field pattern and standing wave : 
pattern measurements of the electromagnetic waves in the interaction region between the horn and the critical layer surface. This detailed information about the lab plasma experiment is expected to result in fairly accurate modeling of the experiment using the SOFTSTEP 2D code developed by Afeyan.

A series of experiments was performed in which the interaction between the reflectometer beam and an externally imposed density fluctuation was constrained to take place within the confines of a wire mesh horn with a long linear taper. Here, clear signs were observed of two distinct interaction regions. The first was located in close proximity to the cutoff layer, while the second was positioned farther down the density gradient. This second response was observed to be progressively delayed in time with increasing wavenumber up to $k=\sim 2 \mathrm{k}_{0}$ consistent with Bragg scattering and never before been observed in the laboratory plasma device. Corroborating evidence for two distinct plasma responses was generated shortly thereafter by the modeling efforts of Cohen and Afeyan (see Fig. 8), who oberved two distinct responses for reflectometry in one-dimensional plasmas similar to that present in the laboratory plasma device.

In an effort to better understand how the two interaction mechanisms function, the original metal horn experiment was then revisited. Here, the increased sensitivity detection systems that were constructed in the first six months of FY95 permitted us to similarly observe two distinct interaction regions in this configuration as well. Efforts were then focused on understanding the reasons why both responses are presently observed while only a single response (located close to the plasma critical surface) was previously observed in the initial studies at UCLA. First indications are that the answer lies in the longer density scale lengths in the present configuration, which was enabled by the addition of a chamber extension to the bottom of the chamber thus lowering the horn position within the chamber. In addition to the longer scale lengths, the plasma exhibits a more linear density profile rather than the largely quadratic profile experimentally observed while at UCLA. Both effects would serve to increase the size of the Bragg response in the newer experiments.

More recently, the single large transmit/receive horn was replaced with a pair of horns (separate transmit and receive) to study how the large, single horn experiments may have been contaminated by the presence of possibly large horn aperture reflections. In particular, one-dimensional computations show that while the critical layer response can be both phase- and amplitude-modulated, the Bragg response should be primarily phasemodulated only. The laboratory plasma experiment, on the other hand, shows that both responses are phase- and amplitude-modulated. As results with the bistatic horns show virtually no change from the single horn experiments, we were forced to conclude : 
that two-dimensional effects were operative in the laboratory plasma experiment that prevented good agreement with one-dimensional theory. New experiments with the large, single horn configuration are aimed at acquiring sufficient diagnostic detail to permit accurate two-dimensional modeling of the experiment using SOFTSTEP 2D.

\section{B. Ultrashort-Pulse Reflectometry Measurements}

Ultrashort-pulse reflectometry (USPR) is under investigation as an advanced diagnostic technique applicable for next generation tokamaks such as ITER. Laboratory tests of the diagnostic in the fall of 1994, using both cylindrical mirrors and wedged targets, showed good agreement with theory. Results from a proof-of-principle seven channel system placed on the CCT tokamak in October 1994 were extremely positive. Figure 13 shows experimental data obtained on CCT from the first set of ultra-short-pulse reflectometry experiments. Significant amplitude and temporal variations of the two detected waveforms, however, were observed. These variations were thought to be largely a function of the high amplitude fluctuations present in the plasma edge that was being probed by the two channel system. Unfortunately, the system at that time was limited to one set of measurements per plasma discharge.

In the past year, the system has been expanded to 8 frequency channels, and is now capable of acquiring USPR time delay data at rates as high as $200 \mathrm{kHz}$ (a total of 4 successive pulses are required for full profile data, permitting a system data acquisition rate of $50 \mathrm{kHz}$ ). The new system was subsequently tested during a 3 day run period on the CCT tokamak at UCLA, and a 3 day run period on the TEXT-U tokamak at the University of Texas at Austin. Although the data from these two series of experiments are still being analyzed, a number of preliminary observations can be made: (i) Variations in the doublepass time-delay USPR data are minimized ( $<0.1 \mathrm{nsec}$ excursions) in steep densitygradient regions (such as in the edge regions and especially in H-mode plasmas), and is extremely large ( $>5 \mathrm{nsec}$ excursions) in the flatter interior regions of the plasma. (ii) Variations in the double-pass time-delay USPR data have been positively identified as originating from large amplitude density fluctuations in the CCT plasma. Direct correlation was observed with sawteeth signals detected via FIR interferometry, and with sawteeth signals detected on a single-channel interferometer system. (iii) Post-detection low-pass filtering was required to obtain accurate and believable density profiles from the USPR data in the presence of large amplitude fluctuations, so long as the USPR channels were sampled at frequencies considerably higher than that of the fluctuations. (iv) The magnitude of the time-delay excursions in the presence of large amplitude fluctuations appears to be related to the filter bandwidth, in particular to the spatial extent of the plasma : 
region over which the reflectometer's signals reflect and fall within the bandwidth of the filter. This conclusion is also borne out by USPR simulations conducted by Cohen. It is expected that this sensitivity would therefore be reduced by the use of narrower filters (70$100 \mathrm{MHz}$ wide vs. the $290-500 \mathrm{MHz}$ wide filters in the present system), and on higher field and/or higher density tokamaks where such filter bandwidths correspond to significantly smaller regions of plasma. The USPR system is presently being modified to incorporate reduced bandwidth filters and a high-speed switch to mask out unwanted port reflections, and will be tested on the GAMMA-10 mirror machine in Tsukuba, Japan in May/June 1996.

\section{Acknowledgments}

We are grateful to Greg Simonson, Tom Casper, Gary Smith, and Ed Williams for helpful suggestions, assistance, interest and support for this activity.

\section{References}

B. B. Afeyan, et al. (1994), Proceedings of the Second IAEA Topical Workshop on Plasma Reflectometry, Princeton, NJ, Jan. 1994.

B. B. Afeyan, A. E. Chou, and B. I. Cohen (1995), Plasma Phys. Control. Fusion 37, 315.

S. Baang, et al. (1990), Rev. Sci. Instrum. 61, 2905.

A. E. Chou, et al. (1992), Rev. Sci. Instrum. 63, 4669.

A. E. Chou, et al. (1995), Rev. Sci. Instrum. 66, 1216.

B. I. Cohen, B. B. Afeyan, A. E. Chou, and N. C. Luhmann, Jr. (1995), Plasma Phys. Control. Fusion 37, 329.

C. W. Domier, et al. (1992), Rev. Sci. Instrum. 63, 4666.

C. W. Domier (1995), Rev. Sci. Instrum. 66, 399.

E. J. Doyle, et al. (1990), Rev. Sci. Instrum. 61, 3016.

J. D. Hansen, G. J. Morales, and R. J. Taylor, Bull. Am. Phys. Soc. 38, 2111 (1993).

A. E. Hubbard, et al. (1987), J. Phys. E 20, 423. I. H. Hutchinson, Plasma Phys. Control. Fusion 34, 1225 (1992).

M. E. Manso, Plasma Phys. Control. Fusion, 35, B141 (1993).

E. Mazzucato (1976), Phys. Rev. Lett. 36, 792.

E. Mazzucato and R. Nazikian (1991), Plasma Phys. Controlled Fusion 33, 261.

S. F. Paul, et al. (1992), Phys. Fluids B 4, 2922.

W. A. Peebles, et al. (1990), Rev. Sci. Instrum. 61, 2905. 
F. Simonet (1985), Rev. Sci. Instrum. 56, 664. 
- The X-mode dispersion relation depends on the local magnetic field intensity. Suppose $B(x)=B_{0}\left[1+\left(x-x_{0}\right) / L_{B}\right]$ for $x>x_{0}$.

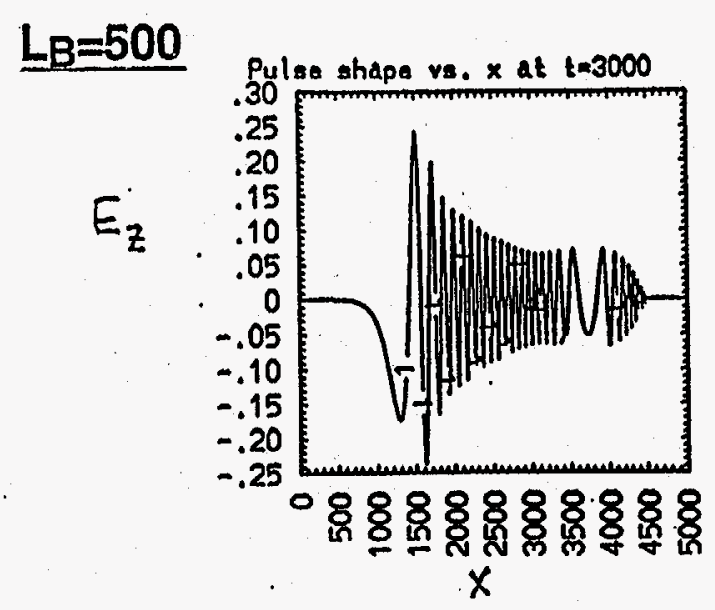

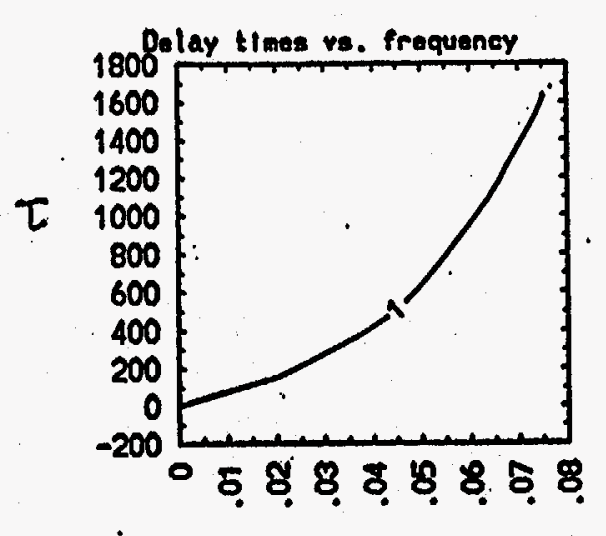

$\omega$

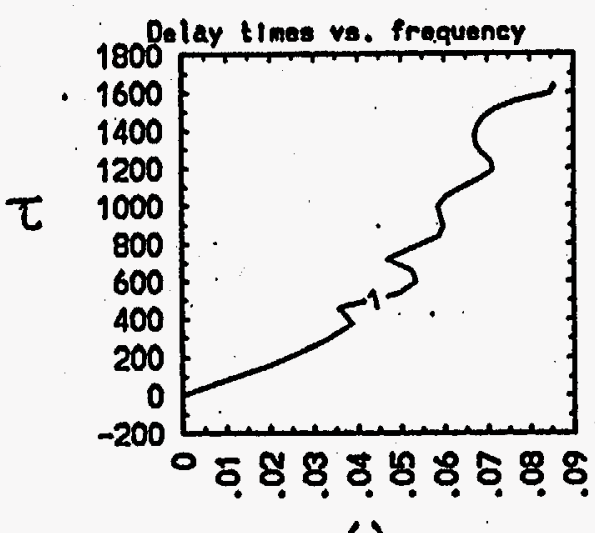

$\omega$

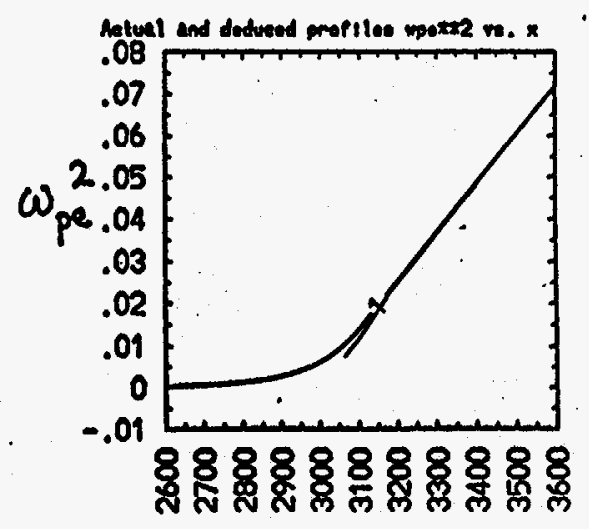

$X$

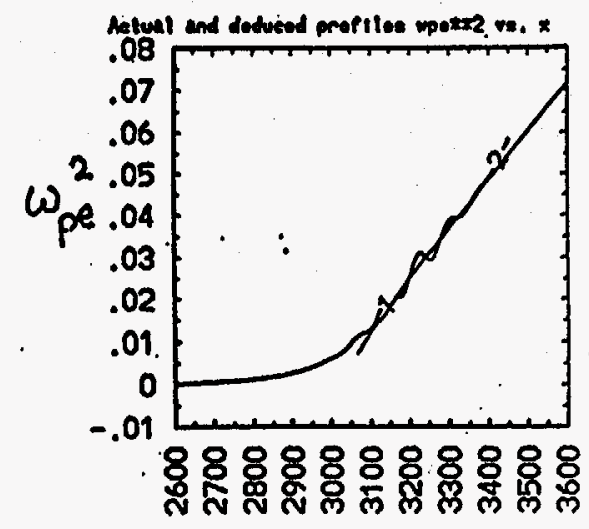

$x$

$$
\omega_{0}=0.75 \pi / \tau_{p} \quad \tau_{p}=20 \quad L_{s}=120 \quad \omega_{c e}(0)=0.35 \quad k_{f}=0.5 \pi / c \tau_{p} \quad x_{w}=L_{s} \quad x_{c}=x_{0}+L_{s} .
$$




\section{D X-Mode Magnetic Field Reconstruction}

- X-modes can be used to reconstruct the magnetic field profile if the plasma density profile is known (e.g., $n_{0}(x)$ deduced from O-mode reflectometry).

$\underline{\delta n / n_{0} \equiv 0, \delta B^{2} / B_{0}{ }^{2} \equiv 0}$

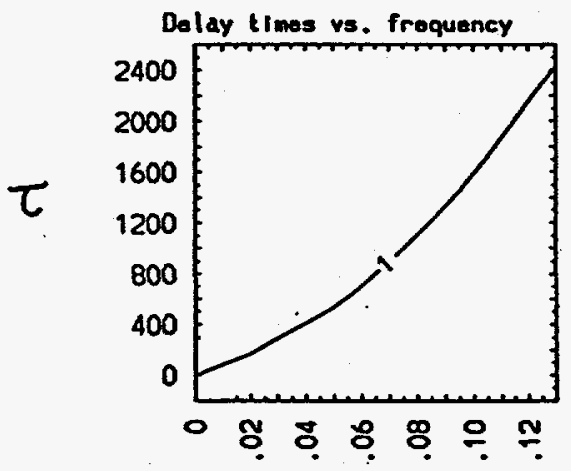

$\omega$

$\underline{\delta n} / n_{0} \equiv 0.2, \delta B^{2} / B_{0}{ }^{2} \equiv 0$

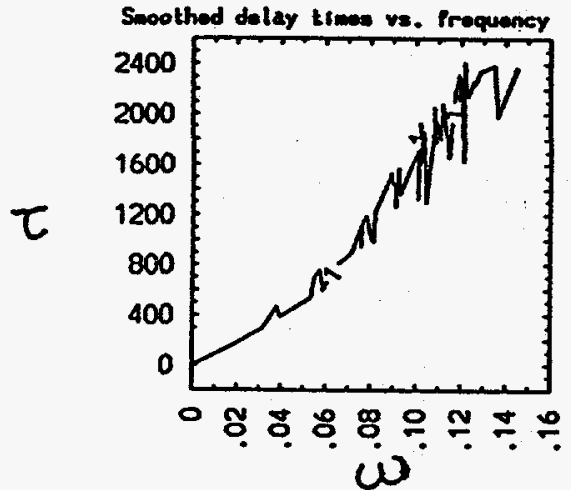

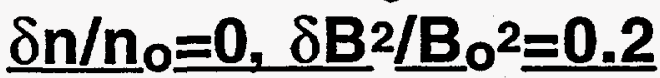

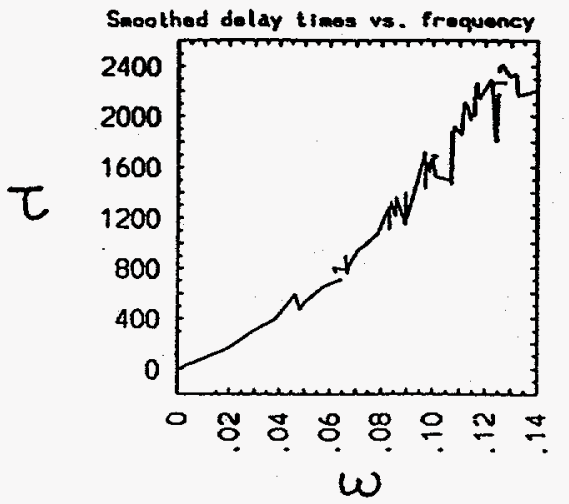

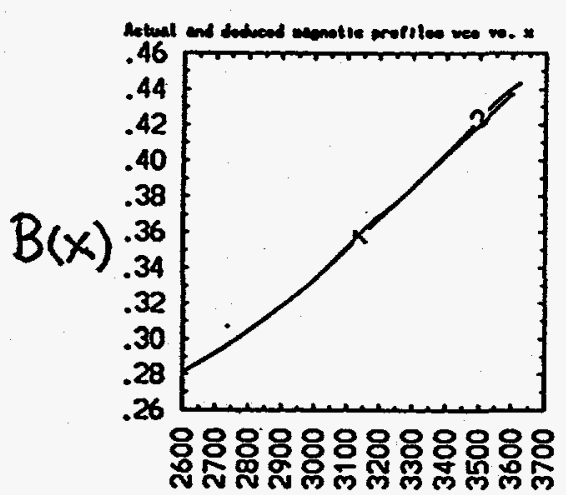

$x$



$X$

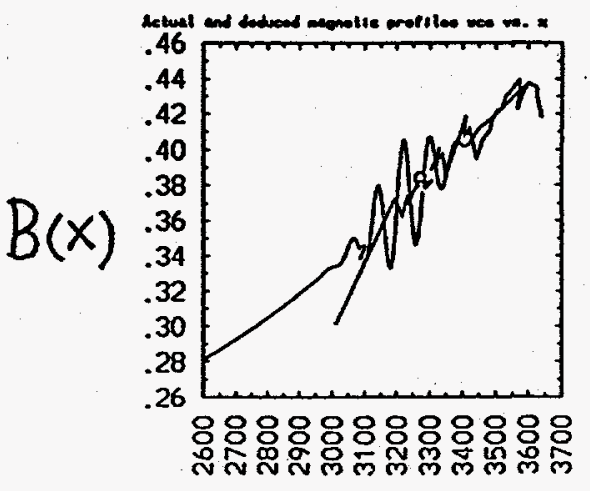

$x$

Figure 2 


\section{Inferring the Fluctuation $\mathbf{k f}$ and Density Scale Length $\mathbf{L}$ s}

- The difference in time delays for signals reflected from Bragg resonance layer and cutoff for a given frequency can be used to infer the wavelength of the fluctuation producing Bragg resonance.

Bragg resonance: $k_{f}=2 k\left(X_{B}\right)=2\left[\omega^{2}-\omega_{p e^{2}}\left(X_{B}\right)\right]^{1 / 2} / c \rightarrow X_{B}=$ Bragg resonance

Difference in time delay $\Delta \tau=2 \int_{x_{C}}^{x_{C}} d x / v_{g}=4\left(L 2-L X_{B}\right)^{1 / 2 / c}$ for a linear density profile $L^{-1}=(d / d x) \ln \omega_{p e^{2}}$ at cutofff $\omega_{p e}=\omega \rightarrow k_{f} / k_{0}=c \Delta \tau(\omega) / 2 L$

- Example:
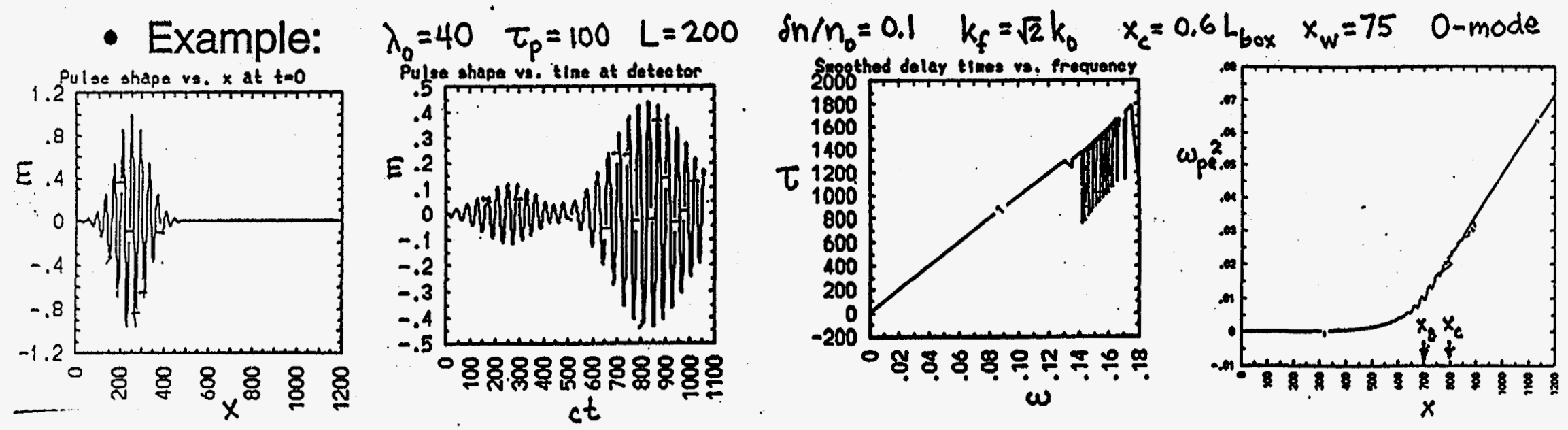

- Using the pulse-determined scale length from the reconstructed density profile, $\mathrm{k}_{f} / \mathrm{K}_{0}=\mathrm{C} \Delta \tau / 2 \mathrm{~L}=600 / 400=1.5 \mathrm{vs}$. actual value 1.414 
- Parameters: $\delta n / n_{0}=0, \lambda_{0}=60, \tau_{x}=30, \tau_{y}=75, L_{s}=100$
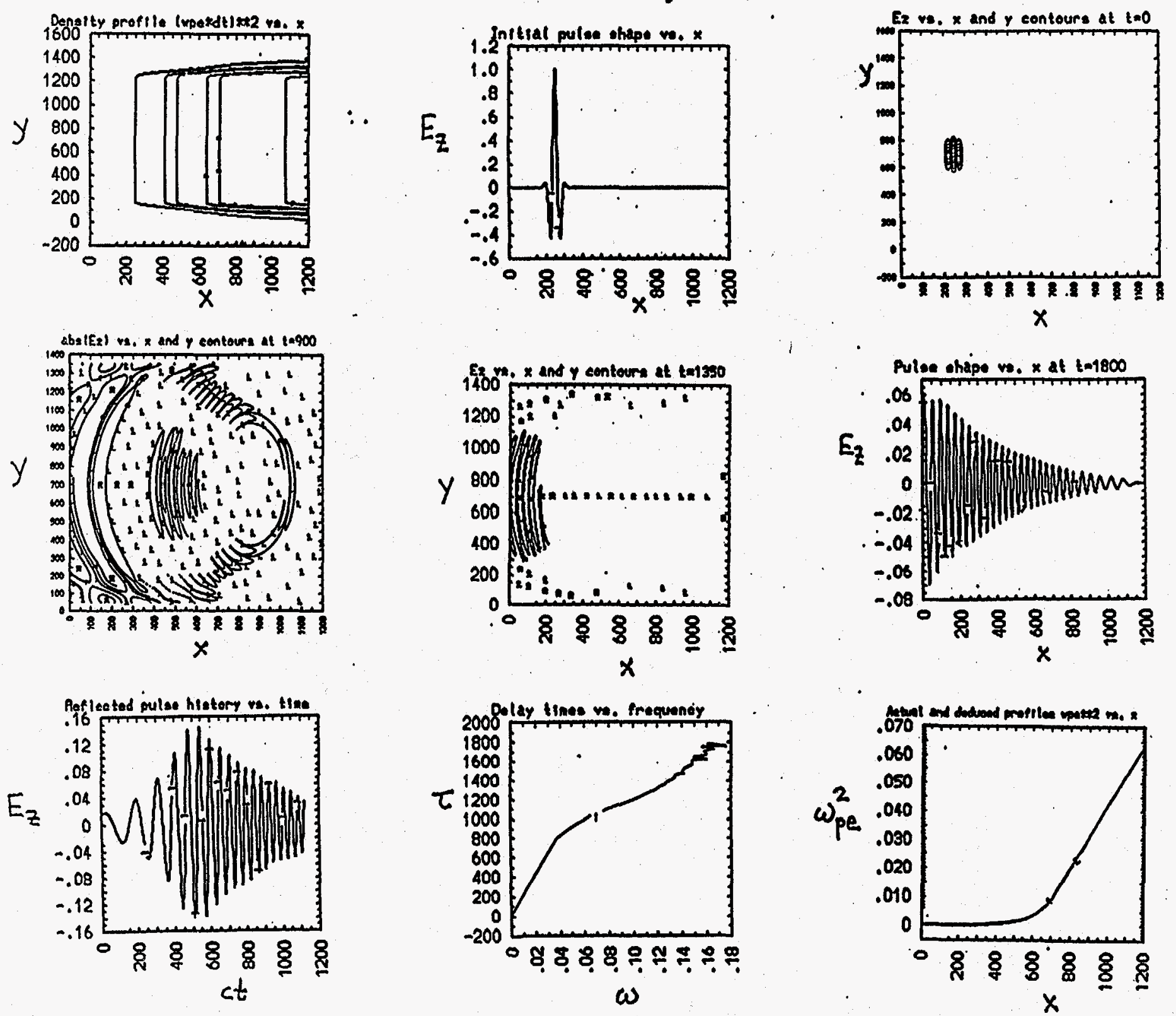


\section{D O-Mode Reconstructions: Spectral \& Zero-Crossing Methods}

- With no density fluctuations and no waveguide present, group delays vs. frequencies determined by either zero-crossing or windowed FFT (spectral method) lead to very good density reconstructions in 2D with O-modes.

\section{zero crossings ( 70 zeros)}
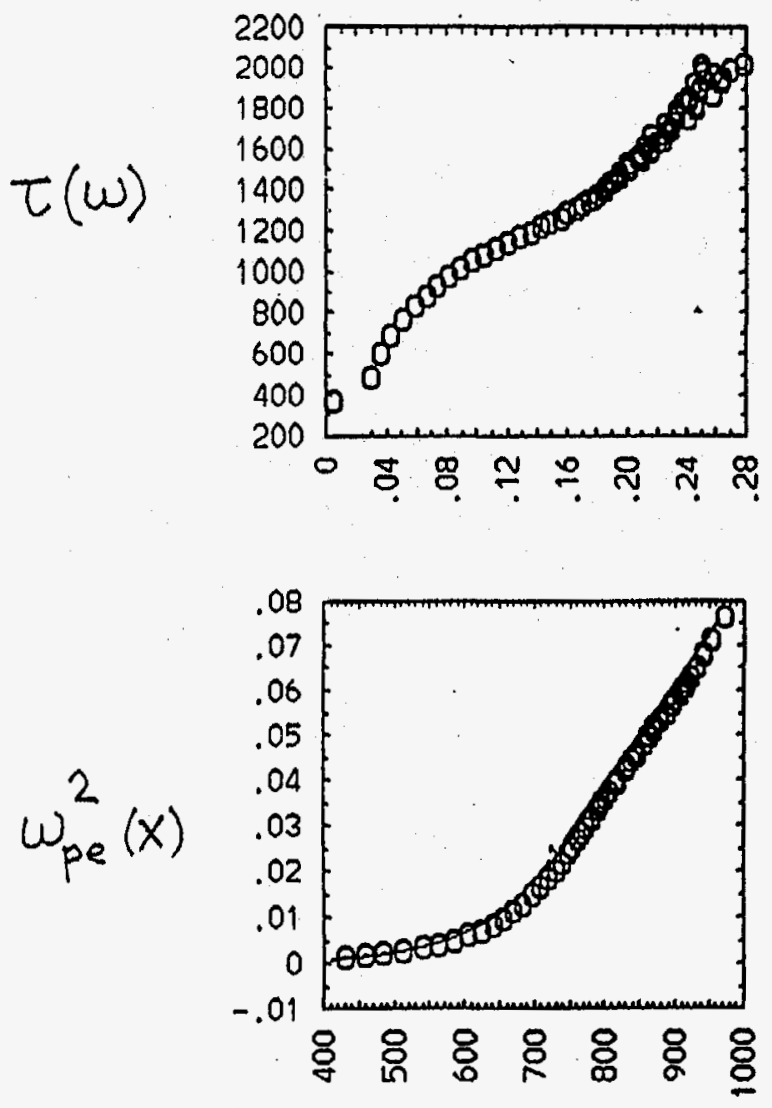
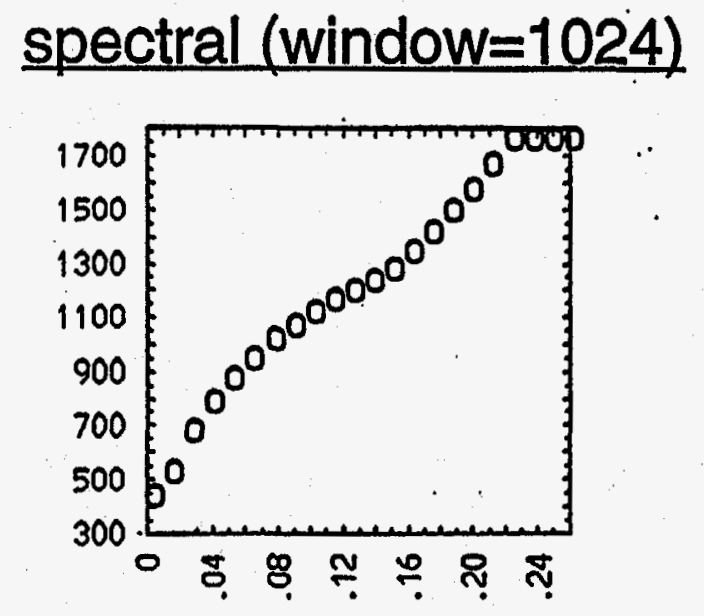

$\omega$



spectral (window $=512$ )
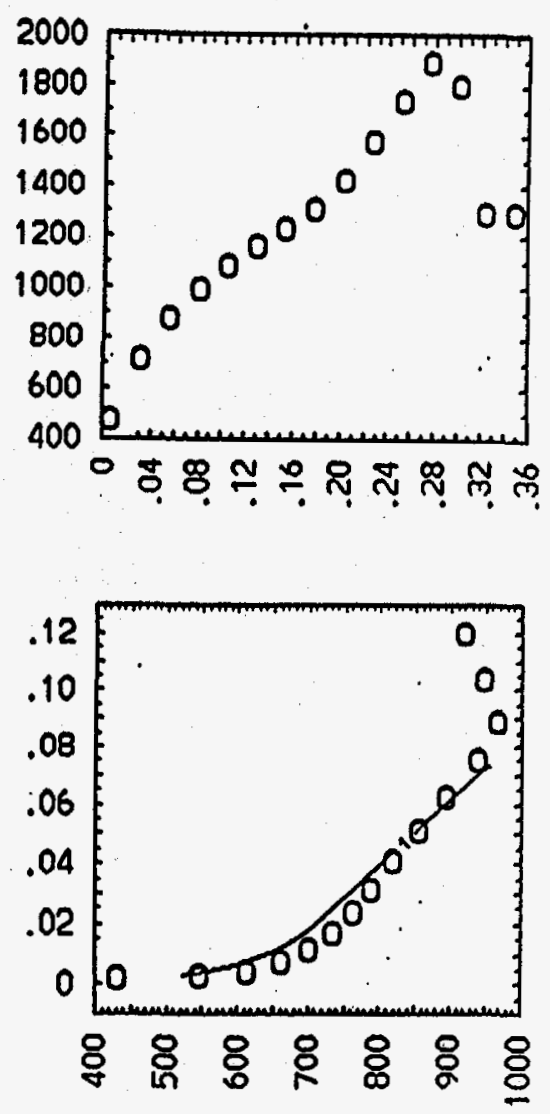


\section{O-Mode Reconstruction of a Stepped Density Profile}

- A stepped $n_{0}(x)$ leads to group delays vs. frequency with a pronounced maximum. Use of spectral analysis ( time-windowed FFT) on reflected electromagnetic field gives sensible group delays which yield a good density reconstruction.
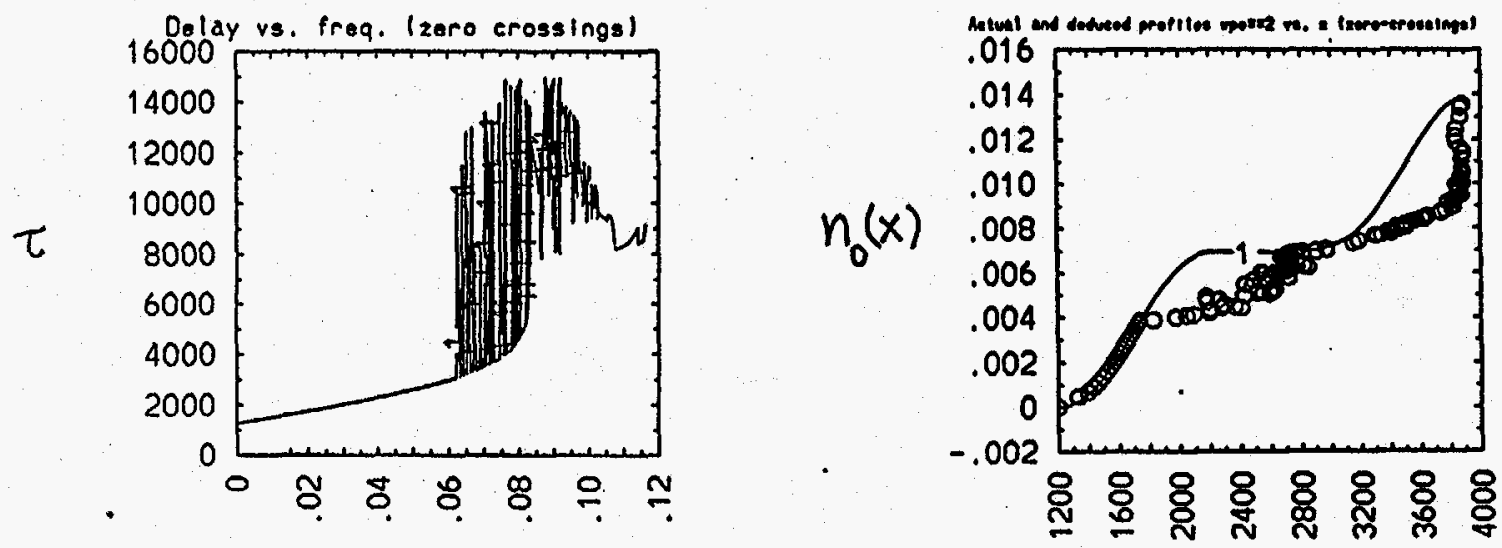

zero crossings

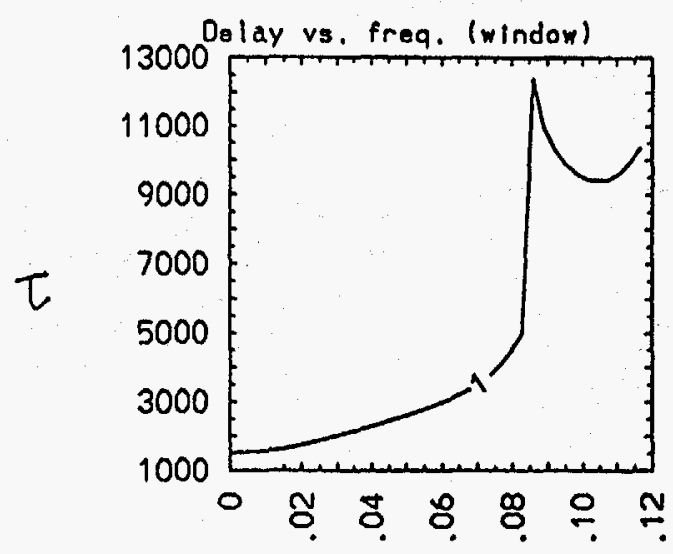

$\omega$

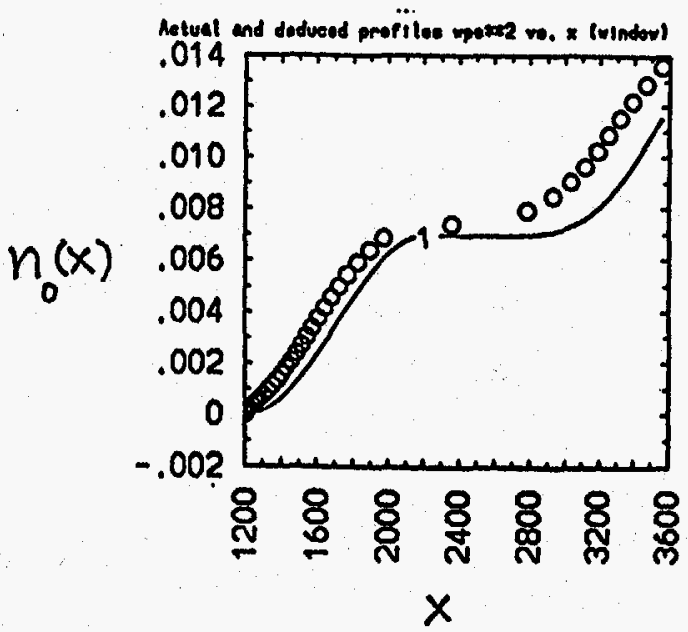

windowed FFT 


\section{D O-Mode Density Reconstructions With a Waveguide}

- 2D O-mode density reconstructions with a waveguide were performed with group delays $\tau(\omega)$ vs. frequency $\omega$ determined by zero-crossings or windowed FFT (spectral) analytically correcting for waveguide dispersion. (Alternatively, a direct waveguide calibration of the waveguide contribution to the group delays could be done with mirror at the end of waveguide.)
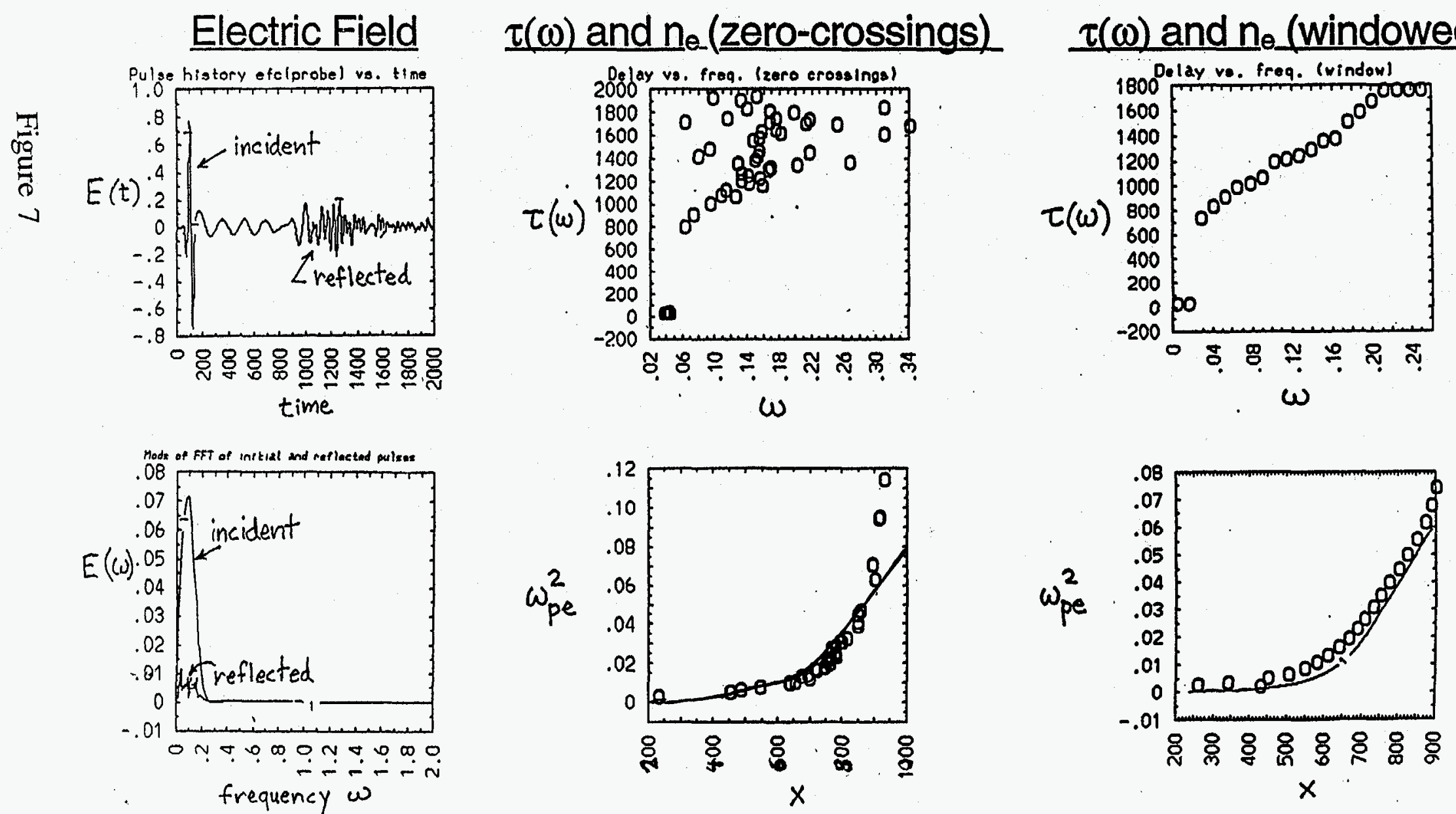
2D O-Mode Reconstructions with Waveguide and Plasma Perturbations

- The windowed FFT (spectral) density reconstructions are very good for a range of density perturbation amplitudes up to $\delta n / n_{0}=0.8$.
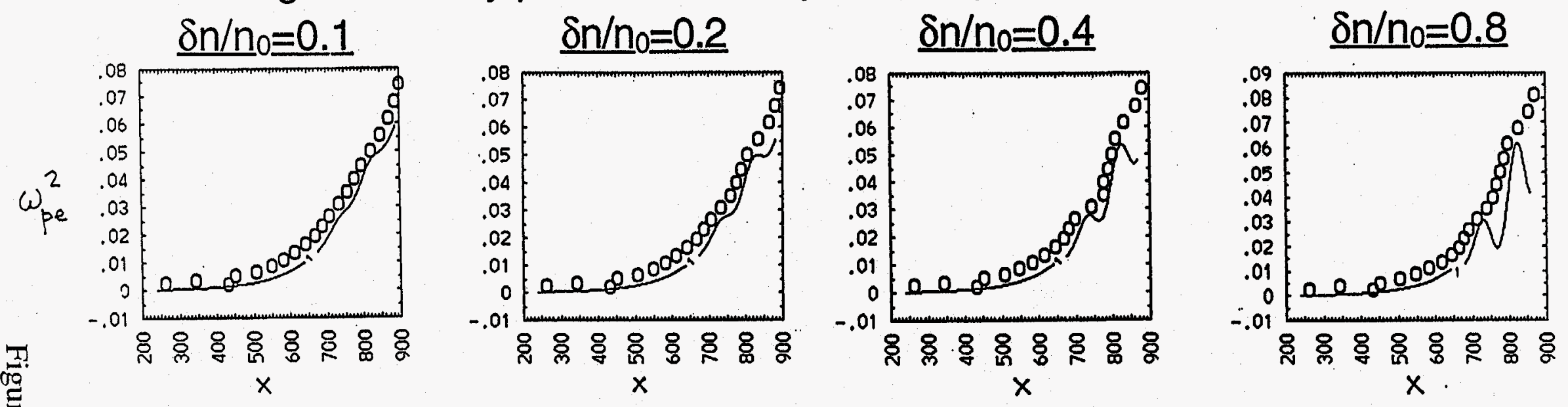

- Bragg resonance effects can be identified in the power spectra vs. time. $\mathrm{k}_{f} / \mathrm{k}_{0}=\mathrm{c} \Delta \tau / 2 \mathrm{~L}_{\text {eff }}$ with $\mathrm{k}_{f} / \mathrm{k}_{0}=1.414$ and $\mathrm{L}_{\text {eff }}=2 \mathrm{~L}_{s}=200$ at Bragg resonance.

wind $=1024$



wind $=256$

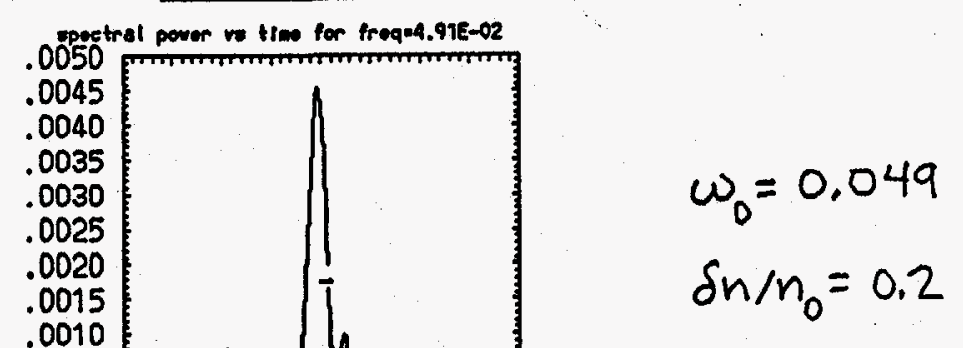

.0005

.000

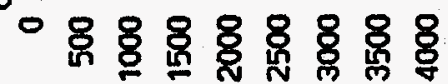

2xtime 
- The first set of ultra-short-pulse experiments by UC Davis Plasma Diagnostics Group on CCT at UCLA have been limited to 8 or fewer frequency channels for the detectors. By varying the FFT window width for a fixed maximum plasma frequency, we could limit the resolution of the determination of group delays vs. frequency and density reconstruction.


window $=1024$
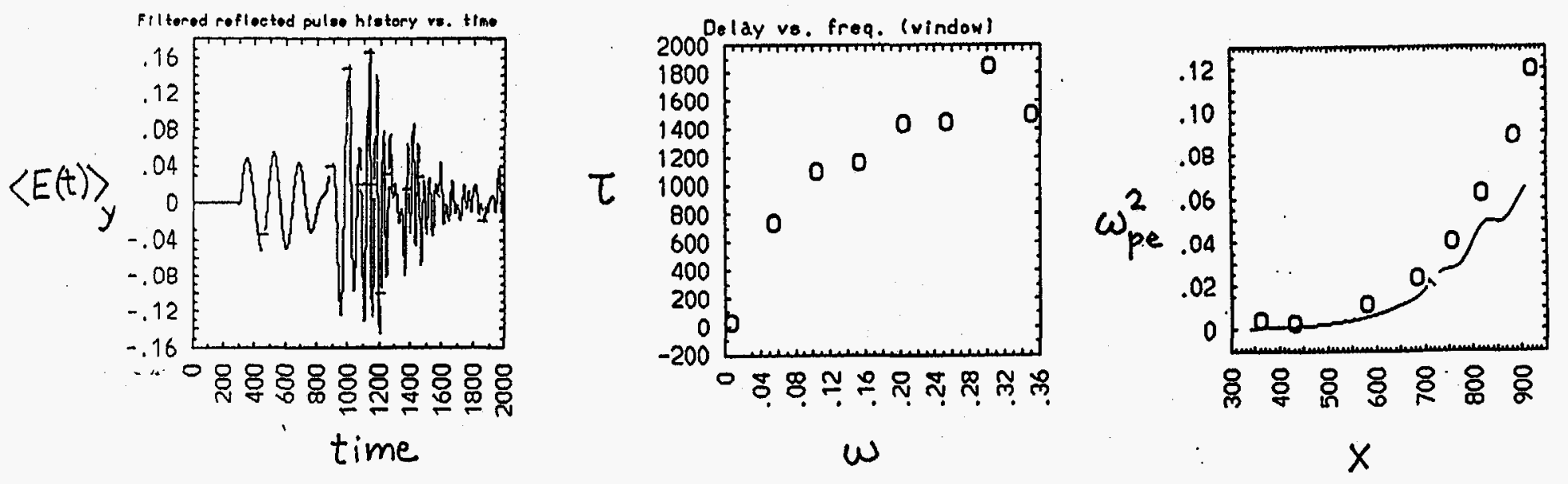

window $=256$

$\omega$ 


\section{Steepest Descent Evaluation of the Scattering Phase Shift: $\bar{k}_{f} \xi_{W} \gg 1$}

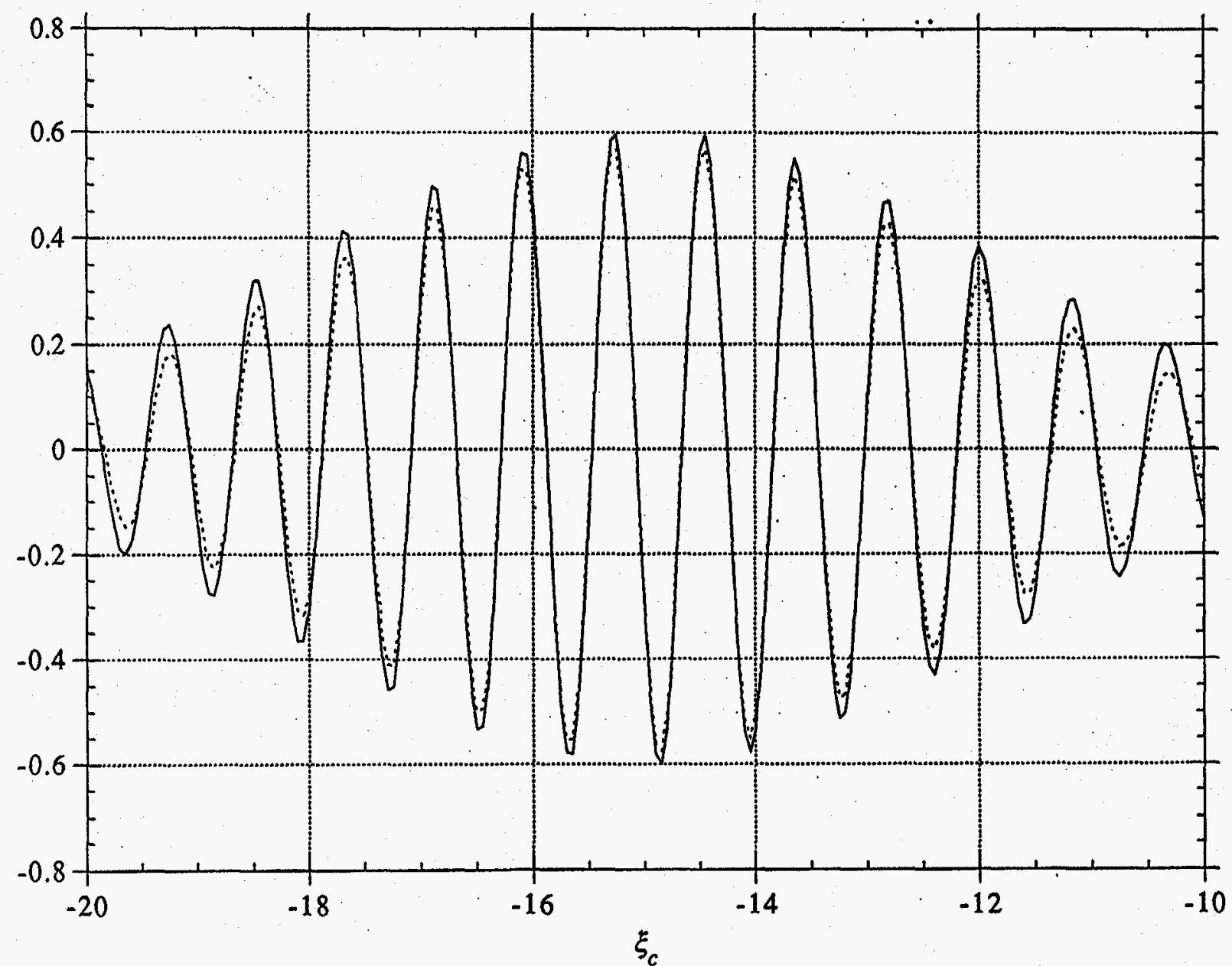




\section{Validity of the Born Approximation}

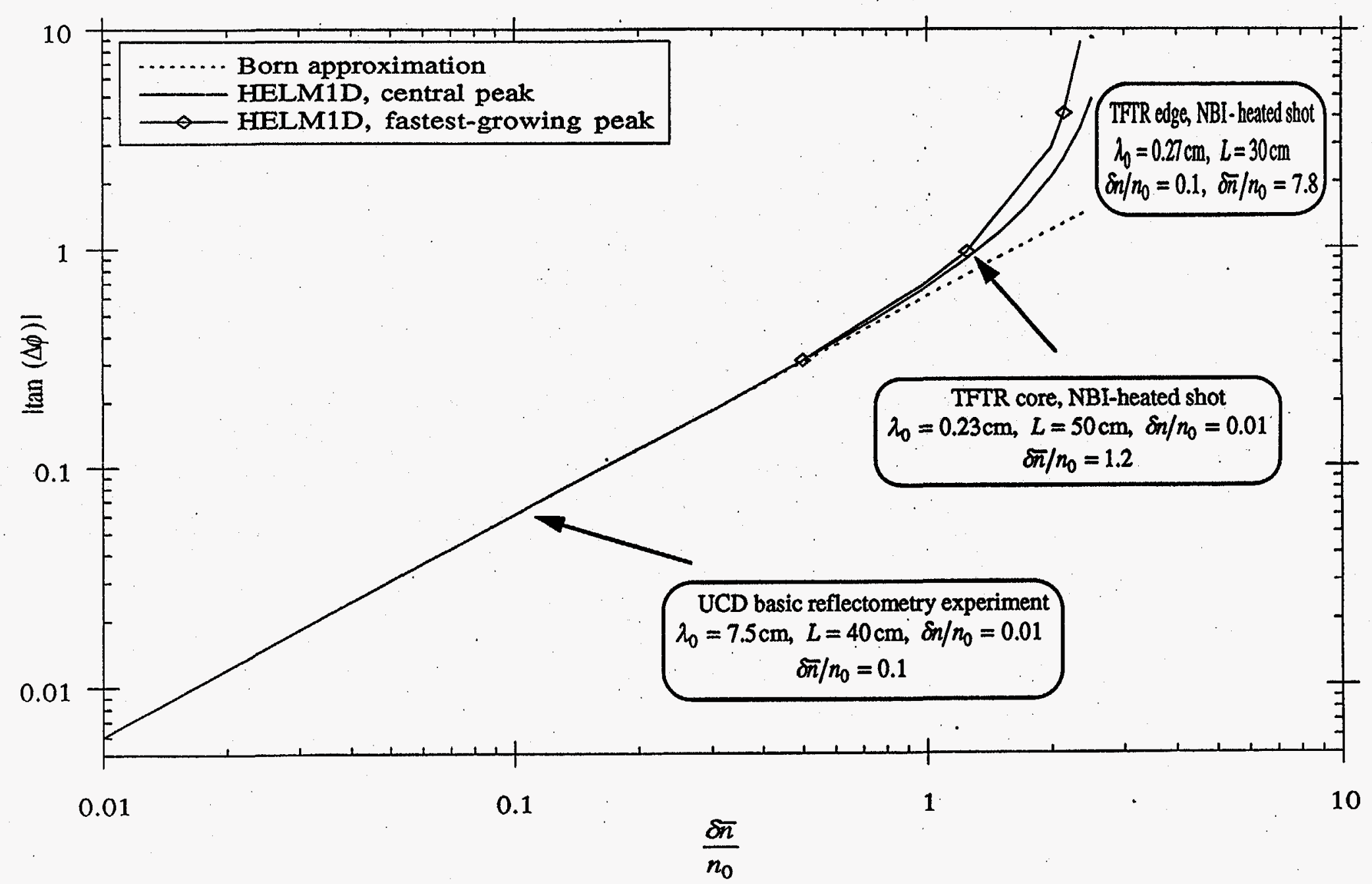

BBA SOFTSTEP 1995. 


\section{Controlled Laboratory Experiment System}

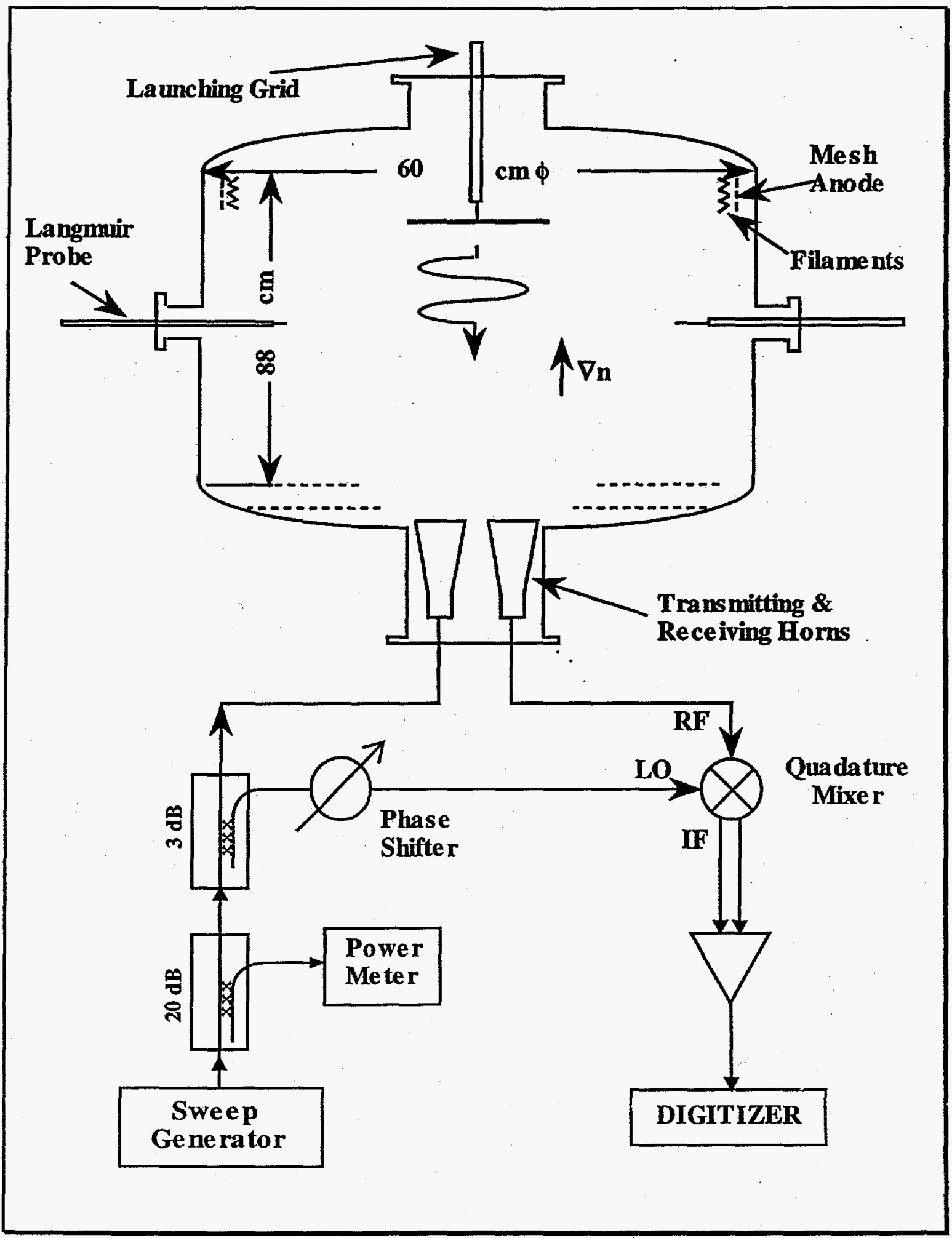

Figure 12 
CCT density profiles by reflectometry: ultrashort-pulse shot \#03

FM shot \#960, average of sweeps 1-4



(C.W. Doniler, UC Davis; A.E. Chou, UCI 